\title{
Apparent and Actual Trajectory Control Depend on the Behavioral Context in Upper Limb Motor Tasks
}

\author{
Tyler Cluff ${ }^{1}$ and Stephen H. Scott ${ }^{1,2,3}$ \\ ${ }^{1}$ Centre for Neuroscience Studies and Departments of ${ }^{2}$ Biomedical and Molecular Sciences and ${ }^{3}$ Medicine, Queen's University, Kingston, Ontario, Canada
}

A central problem in motor neuroscience is to understand how we select, plan, and control motor actions. An influential idea is that the motor system computes and implements a desired limb trajectory, an intermediary control process between the behavioral goal (reach a spatial goal) and motor commands to move the limb. The most compelling evidence for trajectory control is that corrective responses are directed back toward the unperturbed trajectory when the limb is disturbed during movement. However, the idea of trajectory control conflicts with optimal control theories that emphasize goal-directed motor corrections. Here we show that corrective responses in human subjects can deviate back toward the unperturbed trajectory, but these reversals were only present when there were explicit limits on movement time. Our second experiment asked whether trajectory control could be generated if the trajectory was made an explicit goal of the task. Participants countered unexpected loads while reaching to a static goal, tracking a moving target, or maintaining their hand within a visually constrained path to a static goal. Corrective responses were directed back toward the constrained path or to intercept the moving target. However, corrections back to the unperturbed path disappeared when reaching to the static target. Long-latency muscle responses paralleled changes in the behavioral goal in both sets of experiments, but goal-directed responses were delayed by 15-25 ms when tracking the moving goal. Our results show the motor system can behave like a trajectory controller but only if a "desired trajectory" is the goal of the task.

Key words: feedback control; goal-directed corrections; mechanical perturbations; muscle stretch responses; reaching; task constraints

Significance Statement

One of the most influential ideas in motor control is that the motor system computes a "desired trajectory" when reaching to a spatial goal. Here we revisit the experimental paradigm from seminal papers supporting trajectory control to illustrate that corrective responses appear to return to the original trajectory of the limb, but only if there is an imposed timing constraint. We then provide direct evidence that the human motor system can behave like a trajectory controller, and return the limb to its original trajectory when a specified trajectory is the goal of the task. Our results show that the motor system is capable of a spectrum of corrective responses that depend on the behavioral goal of the motor task.

\section{Introduction}

A classic problem in motor control is to understand how the nervous system chooses a limb trajectory to reach a goal among

\footnotetext{
Received March 7, 2015; revised June 22, 2015; accepted July 26, 2015.

Author contributions: T.C. and S.H.S. designed research; T.C. performed research; T.C. and S.H.S. contributed unpublished reagents/analytic tools; T.C. and S.H.S. analyzed data; T.C. and S.H.S. wrote the paper

This work was supported by the Natural Sciences and Engineering Research Council of Canada. S.H.S. was supported by a GSK-CIHR chair in Neuroscience. T.C. was supported by the Natural Sciences and Engineering Research Council of Canada and Banting Postdoctoral Fellowship. We thank Kim Moore and Justin Peterson for expert technical support; and members of the LIMB laboratory for helpful feedback on this manuscript.

S.H.S. is associated with BKIN Technologies, which commercializes the KINARM robotic device used in this study. T.C. declares no competing financial interests.

Correspondence should be addressed to Dr. Stephen H. Scott, Laboratory of Integrative Motor Behaviour, Centre for Neuroscience Studies, 18 Stuart Street, Queen's University, Kingston, Ontario K7L 3N6, Canada. E-mail: steve.scott@queensu.ca.

DOI:10.1523/JNEUROSCI.0902-15.2015

Copyright $\odot 2015$ the authors $\quad 0270-6474 / 15 / 3512465-12 \$ 15.00 / 0$
}

an infinite number of possible paths. Human reaching movements tend to follow relatively smooth, straight hand paths with bell-shaped speed profiles (Morasso, 1981). Further, seminal studies highlight that subjects steer their hand back to its original trajectory when it is mechanically perturbed during movement (Bizzi et al., 1982, 1984; Won and Hogan, 1995). These patterns of limb motion have led to the idea that the motor system computes a desired limb trajectory (Hogan, 1984, 1985; Flash and Hogan, 1985), an intermediary control process between the behavioral goal (reach a spatial target), and the generation of motor commands to move the limb through the desired path to reach the goal.

The idea of trajectory control makes it natural to search for neural correlates of desired limb trajectories. Such patterns of neural activity have been discovered in motor (Georgopoulos et al., 1986) and premotor areas (Caminiti et al., 1991) of cerebral 
cortex. Although correlates of hand motion in M1 have been questioned (Mussa-Ivaldi, 1988; Todorov, 2000; Scott et al., 2001), the assumption that hand trajectories are specified in motor cortex remains popular (Archambault et al., 2011, 2015; Hatsopoulos and Amit, 2012; Dickey et al., 2013).

The principles of optimal feedback control (OFC) have also become influential for interpreting voluntary motor control. The basic approach of OFC is that motor commands are dictated by a cost function that depends on the behavioral goal (Todorov and Jordan, 2002; Scott, 2004; Todorov, 2004; Diedrichsen et al., 2010). An important feature of OFC is the flexible use of sensory feedback where movement errors that detract from attaining the goal are corrected whereas errors that do not interfere with the goal are ignored. This "minimum intervention principle" allows the motor system to minimize the cost of movement while pursuing the behavioral goal in the presence of movement errors (Faisal et al., 2008) or external perturbations (Crevecoeur et al., 2014). OFC emphasizes the link between the task and behavior and predicts that there is no need to control the trajectory if the goal is to reach a spatial target. The predictions of OFC are consistent with the observation that motor corrections are shaped by features of the task, such as the size and orientation of the goal (Nashed et al., 2012; Crevecoeur et al., 2013).

Although models have been developed to meld the concepts of trajectory control and optimal feedback control (Mistry et al., 2013; Kistemaker et al., 2014), the principles of OFC are really at odds with the idea of controlling a specific trajectory to reach a spatial goal. To resolve this issue, we revisit the paradigm from seminal papers supporting trajectory control and demonstrate that task instructions can produce profound differences in behavior. We then perform a second experiment to test whether trajectory control can be generated if the movement path is an explicit goal of the task. Our results indicate that the motor system can behave like a trajectory controller when the limb trajectory is the goal of the task.

\section{Materials and Methods}

Subjects. A total of 30 naive participants (21-53 years of age; 16 males and 14 females) were recruited for one of three experiments. All participants were right-handed and neurologically healthy and had normal or corrected vision. Participants gave written informed consent to protocols approved by the Queen's University Research Ethics Committee and were compensated for their time.

Apparatus and behavioral task. Participants made reaching movements to targets in the horizontal plane while seated with their right arm supported in a robotic exoskeleton. The robotic exoskeleton can apply independent mechanical loads to the shoulder and/or elbow joints (Scott, 1999; Singh and Scott, 2003) (KINARM, BKIN Technologies). Visual targets and hand-aligned feedback were projected into the plane of the participant's hand via a virtual reality display and semisilvered mirror. Direct vision of the arm and hand was blocked with a physical barrier and cloth bib.

In all experiments, participants made goal-directed reaching movements from a start position to an end target. At the start of each trial, the visual display consisted of a 1 -cm-diameter start position that maintained the same limb configuration across participants $(2 \mathrm{~cm}$ diameter in Experiment 2). The location of the participant's hand was displayed as a $0.8-\mathrm{cm}$-diameter white cursor. Trial pacing was self-initiated. Participants began the trial by moving their hand to the start position, and after a brief delay period $(1000 \pm 500 \mathrm{~ms}$, uniformly distributed), were presented with the goal target (diameter $=2 \mathrm{~cm}$; see Fig. $1 A$ ). Task instructions were to reach the goal target and hold this position for $1 \mathrm{~s}$.

Experimental paradigms

Experiment 1A: influence of time constraints on motor corrections during single-joint elbow movements. Subjects $(n=10)$ performed goal-directed elbow movements with their arm supported in a robotic exoskeleton. We physically locked the shoulder at $45^{\circ}\left( \pm 0.1^{\circ}\right)$ relative to the frontal plane by inserting a steel pin into the shoulder joint of the robot (see Fig. $1 \mathrm{~A}$ ). The initial elbow angle was $65^{\circ}$, defined as the external angle of the forearm relative to the upper arm $\left(0^{\circ}\right.$ is full extension $)$. We asked subjects to make $50^{\circ}$ elbow flexion movements (mean target size $=2.81^{\circ}$ elbow motion) under time constraints used to generate moderately fast movements (reach the goal within 600-1000 ms and then hold the target for $1 \mathrm{~s})$. The end of the movement was defined as the first time point when the participant's hand entered and stayed within the target position for $10 \mathrm{~ms}$. Movement times were defined as the time period between the participant's hand leaving the start position and the end of movement. Subjects received post-trial feedback about the timing of their movements. The goal target turned green during the hold period to indicate successful timing. The goal target turned blue (too fast) or red (too slow) if participants did not complete their reach within the time limits. We included a second condition where subjects were instructed to reach the target with no imposed limits on movement time (time-unconstrained task). Participants always received green target feedback (good timing) in this unconstrained time task. Participants performed 120 unperturbed trials and 40 perturbed reaching trials (see below) in each task (320 trials total). Half of our participants $(n=5)$ performed the time-constrained task first, whereas the others performed the time-unconstrained task first $(n=5)$.

Perturbation trials. We measured feedback responses by applying elbow perturbations (randomly interleaved on $25 \%$ of trials) that pushed the subject's hand toward or away from the target (see Fig. $1 A ; \pm 2 \mathrm{Nm}$ step torque with $10 \mathrm{~ms}$ ramp-to-hold profile). Peak shoulder motion during perturbation trials did not exceed $\pm 0.2^{\circ}$. Perturbations were applied at movement onset, which we defined as the time point when the subject's hand left the start position. In the timeconstrained task, participants had to complete their reach within the same time window as their unperturbed movements $(600-1000 \mathrm{~ms})$ and received the same post-trial feedback about movement timing (green target: good timing; red: too fast; blue: too slow). Hand-cursor feedback was always present as pilot work demonstrated that initial feedback corrections and muscle stretch responses were similar regardless of whether visual feedback of the hand's position was provided after perturbation onset (data not shown).

Experiment 1B: influence of time constraints on motor corrections during multijoint reaching movements. To verify that our results generalized beyond single-joint movements, we performed an experiment where we perturbed participants toward (see Fig. $3 A$ ) or away from the goal during multijoint reaching movements (see Fig. $4 A$ ). Subjects $(n=10)$ made 10 $\mathrm{cm}$ reaching movements in two tasks with varying time demands. In the time-constrained task, subjects were instructed to reach the goal target within 600-1000 ms and then hold the target position for $1 \mathrm{~s}$. The goal target turned green to indicate successful timing but turned blue (too fast) or red (too slow) if the subject did not complete their reach within the instructed time. The goal target always turned green in the timeunconstrained task. We measured feedback responses on random trials ( $25 \%$ of trials) by applying endpoint forces that pushed the hand toward or away from the goal (see Figs. $3 A$ and $4 A ; \pm 7 \mathrm{~N}, 10 \mathrm{~ms}$ sigmoidal ramp-to-hold profile). Participants performed 120 unperturbed and 40 perturbed reaching trials with and without time constraints (320 trials total). Trials were divided into 2 blocks separated by a 2 min break. Other aspects of the experiment were identical to Experiment 1A.

Experiment 2: influence of trajectory constraints on motor corrections during multijoint reaching movements. A number of studies have suggested the motor system can engage different control strategies based on the goal of the task. It appears that motor commands preserve the behavioral goal of reaching to or hitting a static target (Todorov and Jordan, 1998, 2002), but there may be circumstances where subjects implement specific hand or limb trajectories (Todorov et al., 1997). Here we disturbed the limb to test how explicit instructions to maintain a specified limb trajectory influence corrective responses during movement. We predicted that corrective responses would direct the hand back to the movement trajectory when it was made an explicit goal of the task.

Subjects $(n=10)$ performed $15 \mathrm{~cm}$ reaching movements in tasks where they were instructed to reach to a static target (static target; see Fig. $5 A$ ) within the allotted time $(600-1000 \mathrm{~ms})$, reach while maintaining 
their hand within a visually specified path to the target (constrained path; see Fig. 5A), or track a target that moved with a bell-shaped velocity profile between the start position and end target (moving target, $1.6 \mathrm{~cm}$ diameter; see Fig. $5 A$ ). The constrained path was implemented using a rectangular area that imposed visual constraints on lateral deviations surrounding a straight path to the goal (see Fig. $5 A$; width $2 \mathrm{~cm}$, length 17 $\mathrm{cm})$. Subjects were instructed to maintain their hand within this constrained movement path, and received color-coded feedback during movement based on whether their hand was inside the constrained path (yellow rectangular target) or outside the path (white rectangular target). In the moving target task, target motion was defined by a time-varying Gaussian velocity profile fit to the participant's forward velocity during unperturbed, time-constrained movements to a static target. Each subject's unperturbed motion patterns were measured during a familiarization task preceding the main experiment. The familiarization task consisted of 40 unperturbed reaching trials to a static target with 10 interleaved perturbation trials (see below). Target motion was fit to the average motion pattern during the last 20 unperturbed reaching trials.

During the main experiment, participants performed 120 unperturbed and 40 perturbed trials in each condition ( 480 trials total). Trials for each task were randomly interleaved and divided into 3 blocks separated by a 2 min break. Subjects were aware of the upcoming task because the moving target or constrained path was present during the preparatory period before movement. The initial shoulder angle was $0^{\circ}$ relative to the frontal plane and the elbow angle was $90^{\circ}$, defined as the external angle of the forearm relative to the upper arm $\left(0^{\circ}\right.$ is full extension $)$.

We measured feedback responses by randomly interleaving perturbations that consisted of equal amounts of shoulder and elbow torque $( \pm 2$ $\mathrm{Nm}$ step torques with $10 \mathrm{~ms}$ sigmoidal ramp-to-hold profile). These combined torque perturbations rapidly displaced the elbow and produced lateral hand motion relative to the goal. This approach allowed for careful matching of movement times, hand kinematics, and perturbations across tasks.

\section{Data recording and analysis}

Kinematics. Angular positions of the shoulder and elbow were sampled at $1 \mathrm{kHz}$ and digitally low-pass filtered (second-order, dual-pass Butterworth, $30 \mathrm{~Hz}$ effective cutoff). Kinematic analysis focused mainly on movement times in Experiments 1A and 1B, which we defined as the first time point the subject entered and stayed within the goal target for 10 consecutive time samples $(10 \mathrm{~ms})$. We also looked at differences in movement times between perturbed and unperturbed movements. Kinematic analysis for Experiment 2 focused on integrated hand path deviations relative to the lateral boundaries of the goal. Integrated hand path deviations were computed for the time period between when the subject's hand left and reentered the lateral boundaries of the goal. We also calculated return times, which we defined as the first time point the subject entered and stayed within the target for 10 consecutive time samples (10 $\mathrm{ms})$. All trials were included in the analysis. Differences in reach kinematics were quantified by comparing individual subject averages for each dependent measure between tasks with single-sided paired $t$ tests. Finally, we examined whether there were learning processes involved in generating goal-directed feedback responses. In Experiments $1 \mathrm{~A}$ and 1B, we compared movement times for each of the first 10 perturbation trials with the grand mean of each task (i.e., criterion value) using one-sampled $t$ tests. Analysis for Experiment 2 focused on the comparison of return times and lateral hand path deviations in each of the first 10 perturbation trials with the grand mean of each task using one-sampled $t$ tests.

\section{Electromyography}

Electromyography signals (EMG) were recorded from the brachioradialis, triceps lateralis, pectoralis major, posterior deltoid, biceps brachii, and triceps longus muscles using bipolar surface electrodes (DE 2.1 Single Differential Electrodes). We focused our analysis on EMG recorded from the monoarticular elbow muscles (brachioradialis, triceps lateral) and shoulder muscles (pectoralis major, posterior deltoid).

We first cleaned the skin surface over each muscle with alcohol to improve signal conductance. Electrodes were then coated with conductive gel and attached to the skin surface over the muscle with double- sided tape. Electrodes were positioned to maximize signals from individual muscles while minimizing crosstalk between muscles. A ground electrode was placed over the lateral malleolus of the ankle or directly over the patella at the knee. EMG signals were amplified (gain = $10^{4}$ ) and sampled at $1 \mathrm{kHz}$ using a Delsys Bagnoli system. EMG signals were then aligned to perturbation onset, bandpass filtered $(20-450 \mathrm{~Hz}$ third-order, dual-pass Butterworth), full-wave rectified, and normalized to the average muscle activity recorded while subjects maintained postural control against $1 \mathrm{Nm}$ extensor (brachioradialis, biceps brachii, pectoralis major) or flexor loads (triceps lateral, triceps longus, posterior deltoid). The postural target was located at the same initial limb configuration as the start positions used in our reaching tasks (for additional details, see Cluff and Scott, 2013).

We quantified muscle stretch responses by aligning EMG to perturbation onset (movement onset) and then subtracting the average EMG recorded in unperturbed trials $(\Delta \mathrm{EMG})$. Analysis of perturbationevoked muscle activity was based on the average muscle activity in predefined time windows (Cluff and Scott, 2013): preperturbation activity (PRE: -50 to $0 \mathrm{~ms}$ ), short latency (SL: $20-45 \mathrm{~ms}$ ), long latency (LL: $45-105 \mathrm{~ms}$ ), and early voluntary time windows (VOL: 105-200 ms). We calculated the average muscle activity in each time window on a trial-bytrial basis and then calculated subjects' average responses in each condition for statistical analysis. The onset of divergence between muscle responses was estimated with receiver operating characteristics analysis (ROC) (Metz, 1978), following procedures described previously (Pruszynski et al., 2008). EMG data were smoothed with a 5 point $(5 \mathrm{~ms})$, bidirectional moving-average filter for display purposes. All dependent measures were calculated from unsmoothed EMG.

\section{Results}

\section{Influence of time constraints on rapid corrective responses}

Experiment 1A: single-joint elbow movements

Subjects made target-directed elbow joint movements with explicit limits on movement time (time-constrained task; 600$1000 \mathrm{~ms}$ ) or without explicit limits on movement time (timeunconstrained task). On random trials, we displaced the subject's hand toward or away from the target by applying loads at the elbow. In the time-constrained task, participants were instructed to complete their reach within the same time period as unperturbed trials (600-1000 ms) and received post-trial feedback on their success.

Figure $1 B$ shows a typical participant's reaching behavior. For this example, we have shown elbow kinematics for individual perturbation trials, as well as averaged elbow trajectories during perturbed and unperturbed movements. We observed brief reversals in elbow motion that commonly began within $250 \mathrm{~ms}$ of perturbation onset, and caused the hand to deviate back toward its unperturbed trajectory (Fig. $1 B$ ). These brief reversals were also evident in group-averaged elbow motion patterns (Fig. 1C). We quantified the tendency to return to the unperturbed trajectory by examining differences in the time taken to reach the target (i.e., movement times) in perturbed and unperturbed trials. Movement times are shown for individual subjects in Figure $1 E$. Movement times were similar between unperturbed and perturbed reaching trials (Fig. $1 E$, blue dots), indicating that subjects were generally able to maintain their movement times and complete trials successfully in the time-constrained task (mean \pm SEM: unperturbed $=872 \pm 107 \mathrm{~ms}$, perturbed $=877 \pm 73 \mathrm{~ms}$, paired $t$ test, $\left.t_{(9)}=0.75, p=0.34\right)$.

Our next step was to identify whether timing features of the task influenced these corrective responses. If there are explicit limits on movement time and the limb is pushed toward the target by a perturbation, one strategy to avoid reaching the target too early $(<600 \mathrm{~ms})$ is to counter the load to slow the limb's motion. The link between the goal, task constraints, and feedback 
A

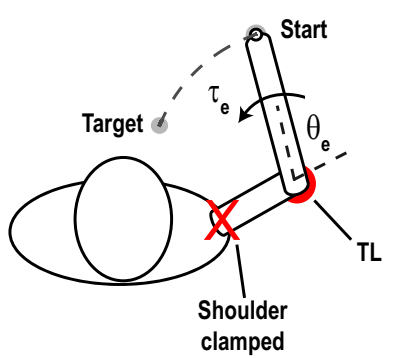

B

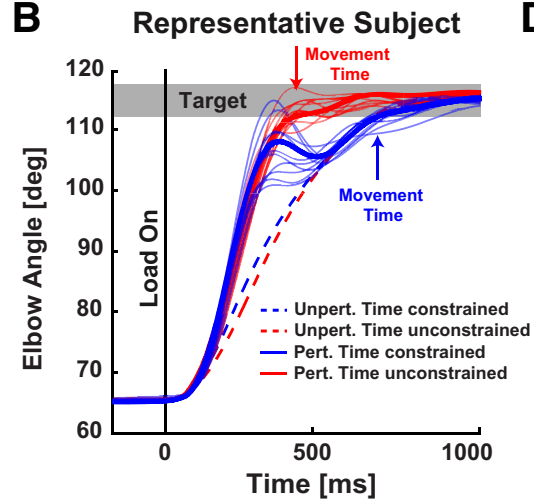

C

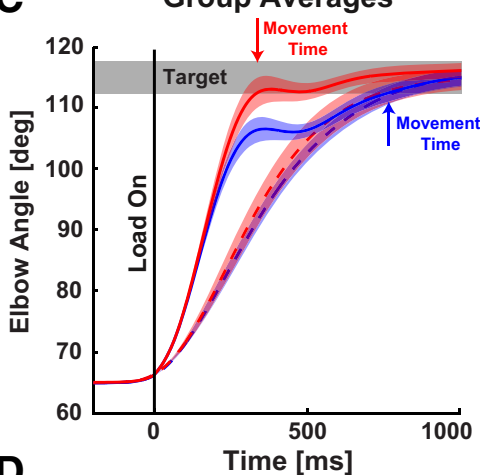

E Individual Subject Averages

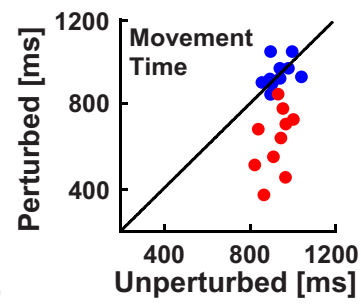

$\mathbf{F}$

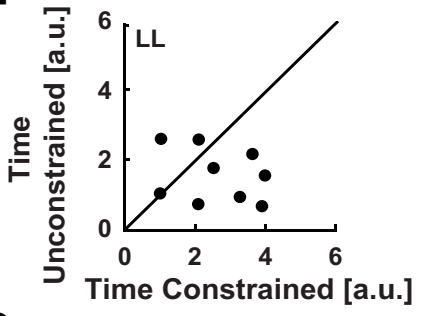

G

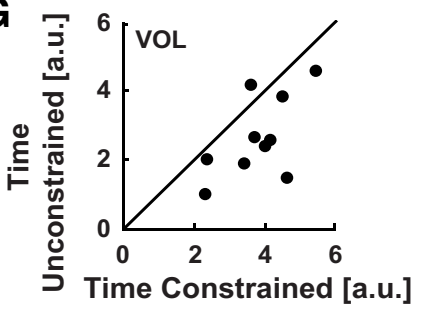

Figure 1. Behavioral task, kinematics, and elbow extensor EMG for single-joint movements with varying time demands. $\boldsymbol{A}$, Target and limb configuration in Experiment $1 \mathrm{~A}$. The limb was occasionally perturbed toward the target with elbow torques, $\tau_{\mathrm{e}}$. B, Elbow motion patterns from a single subject. Thin lines indicate individual trials (10 selected randomly for each condition). Thick lines indicate the elbow's average motion profile. Solid vertical line indicates movement onset, which also corresponds to the onset time of elbow torques during perturbation trials (Load On). Vertical arrows indicate average movement times for perturbed reaching trials in the time-constrained task (blue arrow) and time-unconstrained task (red arrow). $C$, Group data showing average elbow motion profiles. Shaded curve traces represent \pm 1 SEM. Data are plotted in the same format as in $\boldsymbol{B}$. $\boldsymbol{D}$, Average triceps lateral stretch responses $(\Delta$ EMG, $n=10)$. Dashed vertical lines separate time phases of the stretch response. Muscle responses are aligned to perturbation onset (solid vertical line; Load On). Black arrow indicates the onset time of differentiated muscle activity determined using ROC analysis. $\boldsymbol{E}$, Movement times for unperturbed and perturbed trials in the time-constrained task (blue dots) and time-unconstrained task (red dots). Data points indicate individual subject averages. $\boldsymbol{F}, \mathrm{LL}$ muscle stretch responses. $\mathbf{G}, \mathrm{V}$ OL muscle responses. ${ }^{* *} p<0.01 .{ }^{* * *} p<0.001$.

responses is emphasized by OFC (Bryson and Ho, 1975; Todorov and Jordan, 2002). In OFC, task constraints are captured by a cost function that penalizes deviations from the goal, such that varying the time available to complete the task will directly influence movement planning and control (Crevecoeur et al., 2013; Omrani et al., 2013). This link between task and behavior motivated us to investigate how time constraints impact corrective responses. We addressed this question by instructing participants to move to the same goal target without explicit limits on movement time. Subjects always received green target feedback (good timing) after completing movements in the time-unconstrained task.

Figure $1 B$ shows the same representative subject's behavior when we removed the time constraints and disturbed the limb with the same perturbation (red traces). Remarkably, the elbow reversal disappeared when we removed the time constraints, and the subject moved directly to the goal rather than countering the load to return to their unperturbed trajectory (Fig. 1B). Similar results were evident in group-averaged data (Fig. $1 C$ ). Differences in the duration of perturbed reaching trials between tasks were also evident in individual subject averages (Fig. 1E), as well as differences in the time taken by individual subjects to complete perturbed and unperturbed trials in the time-constrained (Fig. $1 E$, blue dots) and time-unconstrained tasks (Fig. 1E, red dots).

We found that subjects reached the goal target much faster when we displaced their limb toward the goal than in unperturbed trials in the time-unconstrained task (mean \pm SEM: un- perturbed $=850 \pm 40 \mathrm{~ms}$, perturbed $=583 \pm 68 \mathrm{~ms}$; paired $t$ test, $\left.t_{(9)}=6.10, p<0.001\right)$. Compared with the time-constrained task, this strategy caused an $\sim 300 \mathrm{~ms}(\sim 30 \%)$ reduction of movement times in perturbed reaching trials in the timeunconstrained task (mean \pm SEM: time constrained $=877 \pm 73$ $\mathrm{ms}$, time unconstrained $=583 \pm 68 \mathrm{~ms}$; paired $t$ test, $t_{(9)}=5.71$, $p<0.001)$. Collectively, our results illustrate that feedback responses are sensitive to the timing demands of the task and can generate motor corrections that look like trajectory control. A key question is how fast these differences in control emerge because the timing of task-dependent responses can provide insight into how they are implemented by the motor system.

We measured how time constraints influenced feedback responses by examining lateral triceps activity during interleaved perturbation trials. Figure $1 D$ summarizes muscle stretch responses evoked by elbow flexor perturbations. In Figure $1 D$, we plotted the group-average response of the lateral triceps muscle obtained by subtracting each subject's perturbation responses from activity recorded during unperturbed trials $(\Delta \mathrm{EMG} \pm$ SEM). Figure 1 also plots the average amplitude of individual subjects' triceps responses in the LL (Fig. $1 F$ ) and VOL time windows (Fig. $1 G$ ), and shows that most subjects increased the amplitude of their LL and VOL muscle responses to counter the load in the time-constrained task. We did not observe significant modulation of muscle responses in the PRE (Fig. $1 D ; t_{(9)}=0.69$, $p=0.94)$ or SL epochs $\left(t_{(9)}=0.38, p=0.36\right)$, signifying that the earliest muscle stretch activity was insensitive to the timing de- 
A
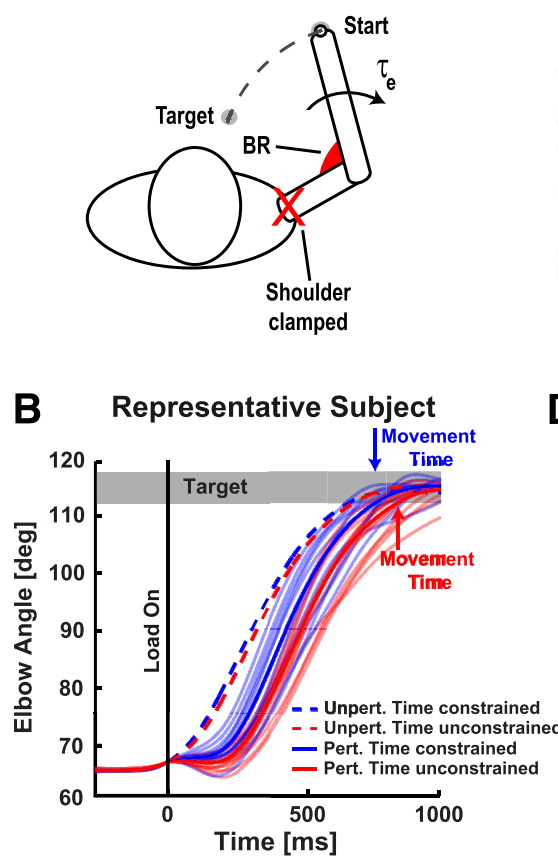

C

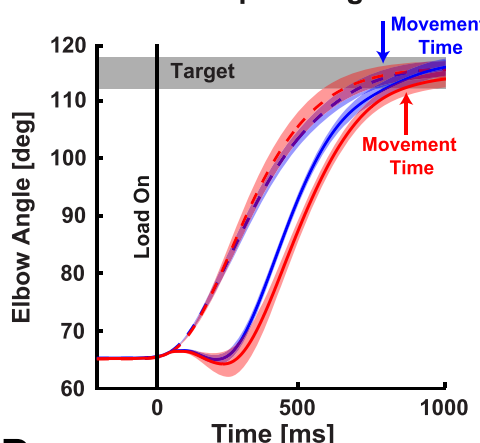

D

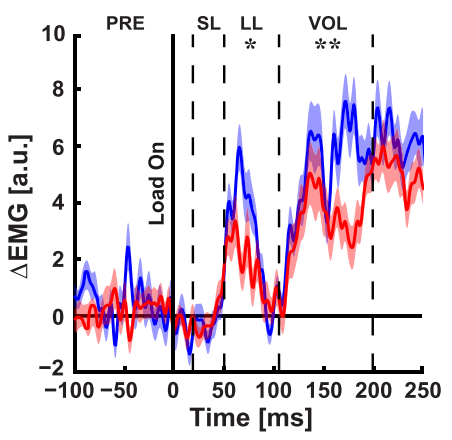

$E_{\text {Individual Subject Averages }}$

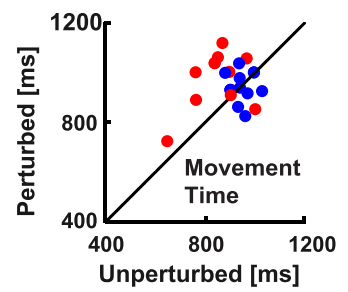

$\mathbf{F}$

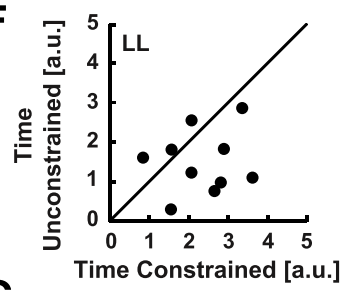

G

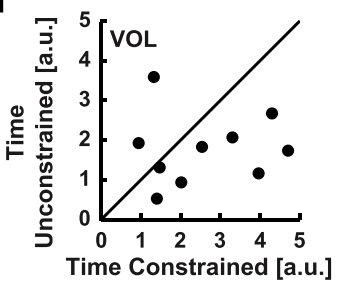

Figure 2. Behavioral task, kinematics, and elbow flexor EMG for single-joint movements with varying time demands. $A$, Target and limb configuration in Experiment 1 A. The limb was occasionally perturbed away from the target with torques that extended the elbow, $\tau_{\mathrm{e}} \cdot \boldsymbol{B}$, Elbow motion patterns from a typical subject. Thin lines indicate motion in individual trials (10 trials selected randomly for each task). Thick lines indicate the average motion profile. Vertical arrows indicate averaged movement times for perturbed reaching trials in the time-constrained task (blue arrow) and time-unconstrained task (red arrow). C, Group data showing average elbow motion profiles $(n=10)$. Data are plotted in the same format as in $\boldsymbol{B}$. D, Group data $(n=10)$ showing brachioradialis responses to elbow extensor perturbations. Data are aligned to perturbation onset (solid vertical line; Load On). Shaded regions represent \pm 1 SEM. $E$, Movement times for unperturbed and perturbed trials in the time-constrained task (blue dots) and time-unconstrained task (red dots). Data points indicate individual subject averages. $F$, LL muscle responses. $G, V O L$ muscle responses. ${ }^{*} p<0.05 .{ }^{* *} p<0.01$.

mands of the task. Removal of the time constraints caused a reduction in triceps responses in the LL and VOL time windows (LL: $t_{(9)}=4.32, p<0.01$; VOL: $t_{(9)}=3.37, p<0.01$ ). ROC analysis indicated that goal-directed muscle responses began in the LL time window ( $\sim 65 \mathrm{~ms}$ after perturbation; range $52-80$ $\mathrm{ms})$. Collectively, our results show the amplitude of LL and VOL stretch responses paralleled timing features of the behavioral task.

Figure 2 plots behavioral results and brachioradialis EMG when a perturbation extended the elbow at movement onset. Again, we found feedback responses that resembled trajectory control when we imposed time limits on movement. This included a faster reversal in elbow motion that allowed participants to return to their unperturbed motion path and complete their reach on time. This effect was evident in representative subject behavior (Fig. 2B), group-average responses (Fig. 2C), and individual subject averages (Fig. $2 E$ ). We found a corresponding increase in movement times when the limb was perturbed in the time-unconstrained task (Fig. 2E, red dots) compared with timeconstrained task (Fig. $2 E$, blue dots; $t_{(9)}=2.39, p<0.05$ ). Whereas subjects took longer to reach the target in perturbed relative to unperturbed trials in the time-unconstrained task $\left.t_{(9)}=3.28, p<0.01\right)$, they maintained similar movement times in perturbed and unperturbed trials in the time-constrained task $\left(t_{(9)}=1.45, p>0.05\right)$.

We contrasted the amplitude of muscle stretch responses to further understand how altered time demands impact behavior. Figure 2 plots group-average muscle responses (Fig. 2D), as well as individual subjects' average muscle responses in the LL (Fig.
$2 F$ ) and VOL time windows (Fig. 2G). Again, we found that the amplitude of most subjects' LL and VOL responses was larger in the time-constrained task. We observed an increase in brachioradialis muscle activity in the time-constrained task compared with the time-unconstrained task, which began in the LL time window (Fig. 2F; $t_{(9)}=1.99, p<0.05$ ) and extended to the early voluntary response (VOL, Fig. $2 G ; t_{(9)}=3.07, p<0.01$ ). Altered timing demands did not significantly influence background muscle activity (PRE: $t_{(9)}=0.38, p>0.05$ ) or SL spinal responses $\left(t_{(9)}=1.62, p>0.05\right)$.

We next examined the time course of behavioral responses to identify whether learning processes were involved in generating goal-directed corrections. In the time-constrained task, subjects moved to the target too quickly in the first flexor perturbation trial $\left(t_{(9)}=-2.72, p<0.05\right)$ and too slowly when the extensor load pushed their hand away from the target $t_{(9)}=2.32, p<$ $0.05)$. Corrective responses were modified by the second perturbation trial to counter the load and move to the target on time (Trials $2-10$, flexion perturbations: all $p>0.14$; extension perturbations: all $p>0.12$ ). In the time-unconstrained task, there was a trend toward subjects taking more time to reach the target during the first flexor perturbation trial (hand pushed toward the target) and less time to reach the target during the first extensor perturbation trial (hand pushed away from the target). These trends did not reach statistical significance (Trials 1-10; flexion perturbations: all $p>0.12$; extension perturbations: all $p>0.13$ ). Collectively, our results demonstrate that subjects required at most one trial to alter their behavior for the timing demands of the task. 
A
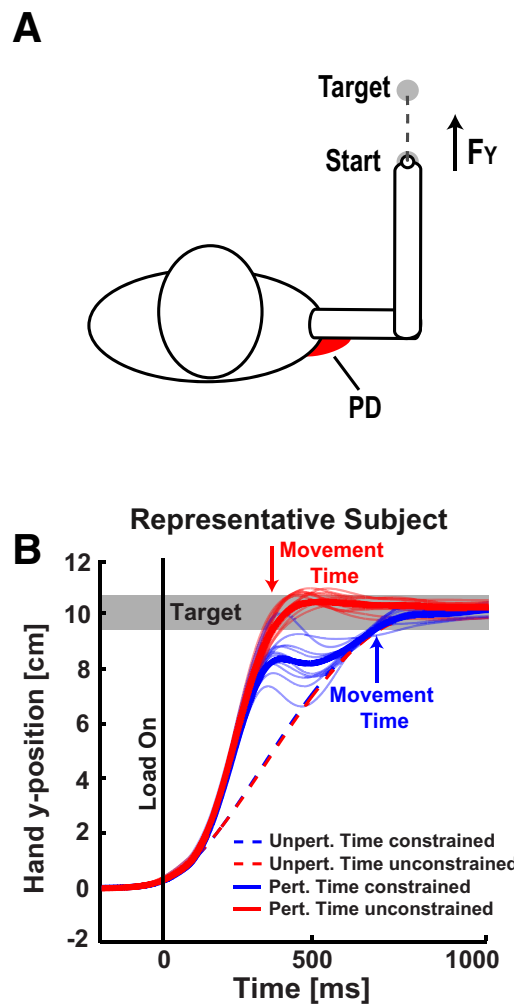

C
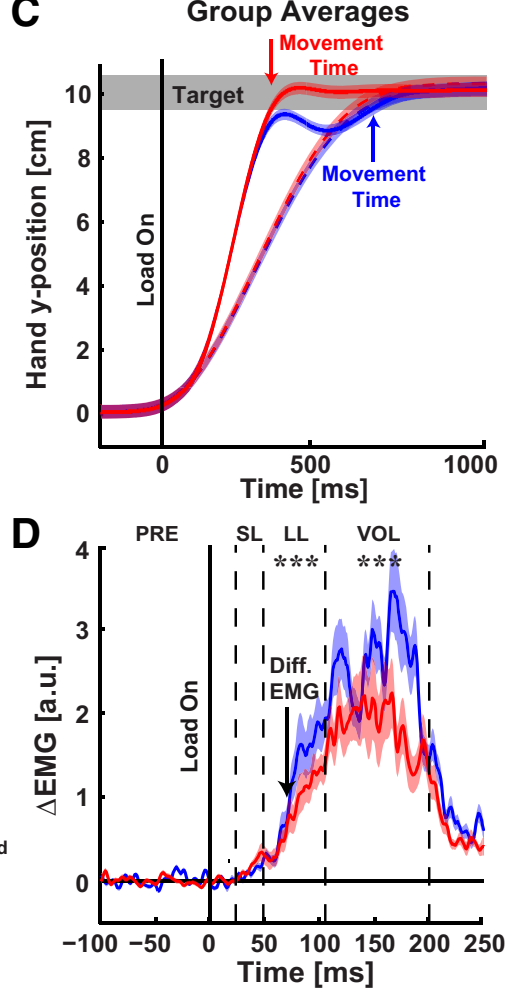

E Individual Subject Averages

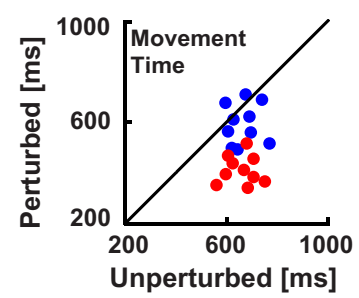

$\mathbf{F}$

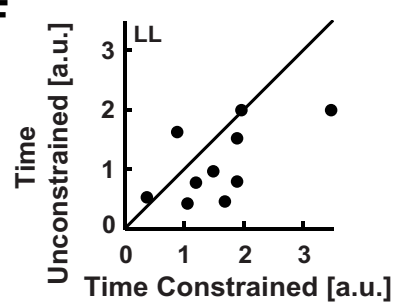

G

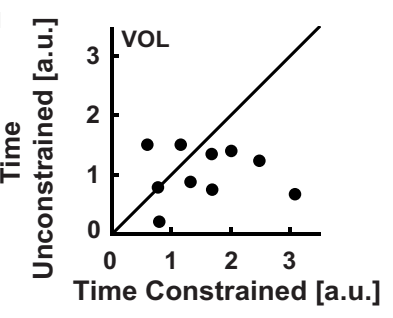

Figure 3. Task, behavior, and shoulder extensor EMG for multijoint movements with varying time demands. $\boldsymbol{A}$, Target and limb configuration in Experiment $1 \mathrm{~B}$. The limb was occasionally perturbed toward the target with forces applied at the hand (Fy). $\boldsymbol{B}$, Typical subject data showing hand motion in the forward direction. Thin lines indicate individual trials. Thick lines indicate average hand motion. Vertical arrows indicate the subject's average movement times in the time-constrained task (blue arrow) and time-unconstrained task (red arrow). $\boldsymbol{C}$, Group-average ( $n=10$ ) forward hand motion. Shaded curve traces represent \pm 1 SEM. Vertical arrows indicate group-average movement times. Data are plotted in the same format as in $\boldsymbol{B}$. $\boldsymbol{D}$, Posterior deltoid stretch responses $(n=10)$. Data are aligned to perturbation onset (solid vertical line). Black arrow indicates the onset time of differentiated muscle activity determined using ROC analysis. $\boldsymbol{E}$, Movement times for unperturbed and perturbed trials in the time-constrained (blue dots) and time-unconstrained tasks (red dots). Data points indicate individual subject averages. $\boldsymbol{F}$, $\mathbf{L}$ muscle stretch responses. $\boldsymbol{G}$, V0 L muscle stretch responses. ${ }^{* *} p<0.001$.

\section{Experiment 1B: multijoint reaching movements}

To verify that our results generalized beyond single-joint movements, we performed an experiment (Experiment 1B) in which we perturbed participants toward the target during multijoint reaching movements (Fig. $3 A$ ). We again found that a simple timing constraint elicited feedback responses that resemble trajectory control. Figure $3 B$ presents forward hand motion from a representative subject during unperturbed and perturbed reaching trials. We observed a brief reversal in hand motion when the limb was perturbed toward the target in the time-constrained task. This brief reversal allowed the participant to slow the limb's motion and complete their reach within the instructed time (Fig. 3B). Brief reversals in hand motion were also evident in group-averaged responses in the time-constrained task, leading to a clear separation of movement times when the limb was perturbed with and without explicit time constraints (Fig. $3 C$ ). Whereas perturbed reaching trials tended to be shorter than unperturbed trials in the time-unconstrained task (Fig. 3E, red dots), most subjects maintained similar movement times in perturbed and unperturbed trials in the time-constrained task (Fig. 3E, blue dots). As we saw in Experiment 1A, the hand reversal disappeared when we removed the time constraints, causing an $\sim 230 \mathrm{~ms}$ $(\sim 35 \%)$ reduction in movement times when the limb was perturbed in the time-unconstrained task compared with the time-constrained task (mean \pm SEM: time constrained $=$ $655 \pm 43 \mathrm{~ms}$, time unconstrained $=428 \pm 39 \mathrm{~ms} ; t_{(9)}=3.06$, $p<0.01)$.
We analyzed the EMG of the shoulder muscles in response to identical perturbations applied with or without time constraints. Figure 3 shows the average posterior deltoid response (Fig. 3D; shoulder extensor), as well as the amplitude of individual subjects' posterior deltoid activity in the LL (Fig. $3 F$ ) and VOL time windows (Fig. 3G). Posterior deltoid responses were larger in the time-constrained task in the LL and VOL time windows (LL: Fig. $3 F ; t_{(9)}=3.24, p<0.001$; VOL: Fig. $\left.3 G ; t_{(9)}=5.18, p<0.001\right)$. There were no significant differences in muscle stretch activity in the PRE or SL epochs (PRE: $t_{(9)}=0.46, p>0.05$; SL: $t_{(9)}=0.83$, $p>0.05)$. ROC analysis indicated that differential muscle responses began in the LL time window $\sim 60 \mathrm{~ms}$ after perturbation onset (range 52-75 ms).

Figure 4 plots behavioral results and pectoralis major EMG when perturbations forced the hand away from the goal at movement onset. We again found differential perturbation responses that depended on the timing demands of the task. Figure $4 B$ plots forward hand motion patterns from the same exemplar subject as Figure $3 B$. We observed earlier reversals in hand motion in the time-constrained task. Similar results are evident in group data (Fig. 4C), as well as individual subjects' average movement times (Fig. 4E). Subjects typically caught up to their unperturbed trajectory and maintained similar movement times in perturbed and unperturbed trials in the time-constrained task (Fig. 4E, blue dots; $\left.t_{(9)}=0.69, p=0.99\right)$. In contrast, the same subjects showed a pronounced increase in the length of perturbed relative to unperturbed trials in the time-unconstrained task (Fig. 4E, red dots; 
A

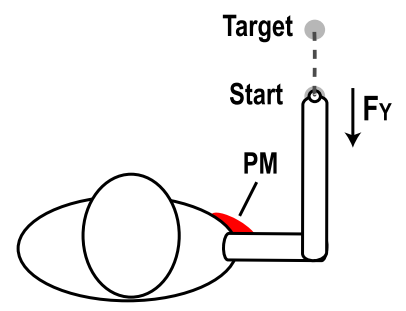

B

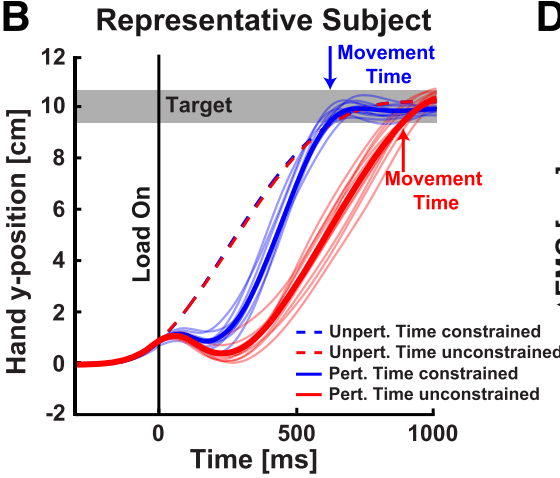

C

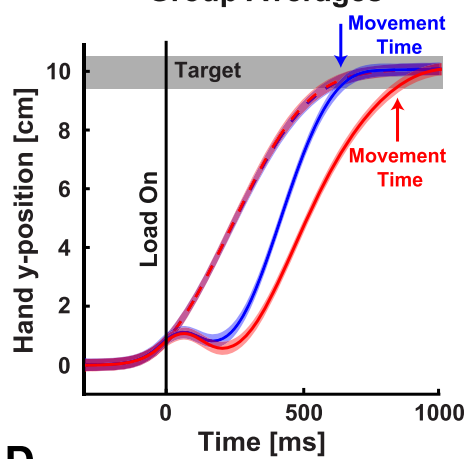

E Individual Subject Averages

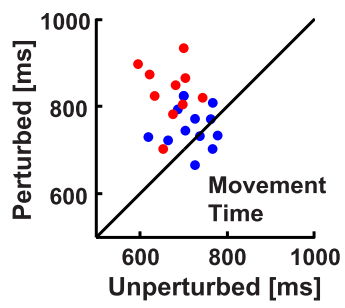

$F$

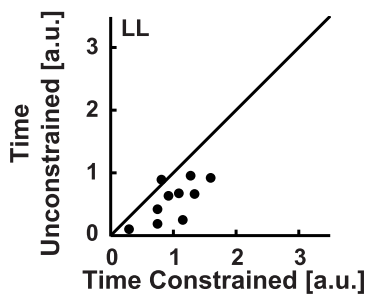

G

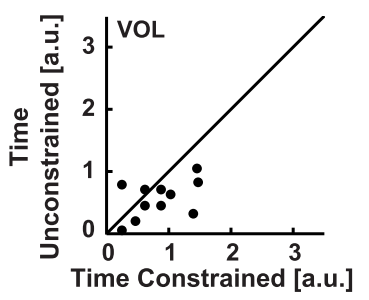

Figure 4. Behavioral task, kinematics, and shoulder flexor EMG for multijoint movements with varying time demands. $\boldsymbol{A}$, Target and limb configuration in Experiment $1 \mathrm{~B}$. The limb was occasionally perturbed away from the target with forces applied at the hand (Fy). B, Typical subject data showing forward hand motion. Thin lines indicate individual trials (10 trials sampled randomly for each condition). Thick lines indicate average motion. Data are aligned to movement onset, which corresponds to perturbation onset (solid vertical line; Load On). Vertical arrows indicate the subject's average movement times in the time-constrained task (blue arrow) and time-unconstrained task (red arrow). C, Group-average hand motion patterns ( $n=10)$. Shaded curve traces represent \pm 1 SEM. Vertical arrows indicate average movement times. Data are plotted in the same format as in $\boldsymbol{B}$. D, Pectoralis major stretch responses $(n=10)$. Data aligned to perturbation onset (solid vertical line). Dashed vertical lines separate time phases of the muscle response. $\boldsymbol{E}$, Movement times for unperturbed and perturbed reaching trials in the time-constrained (blue) and time-unconstrained task (red). Data points correspond to individual subject averages. $\boldsymbol{F}$, LL muscle stretch responses. G, VOL muscle stretch responses. ${ }^{* *} p<0.01 .{ }^{* * *} p<0.001$.

$\left.t_{(9)}=18.7, p<0.001\right)$. Finally, perturbed reaching trials in the time-unconstrained task were longer than the time-constrained task $\left(t_{(9)}=5.35, p<0.001\right)$.

Figure $4 D$ plots the average perturbation-induced activity of the pectoralis major muscle $(\Delta \mathrm{EMG}, n=10)$, as well as the amplitude of individual subjects' LL (Fig. $4 F$ ) and VOL muscle responses (Fig. 4G). In agreement with the behavioral results, we observed an increase in pectoralis major responses in the LL (Fig. $\left.4 F ; t_{(9)}=3.01, p<0.05\right)$ and VOL time windows in the timeconstrained task (Fig. $4 G ; t_{(9)}=5.12, p<0.01$ ). Muscle activity in the PRE and SL time windows was not significantly affected by the timing demands of the task (all $t_{(9)}<1.70, p>0.05$ ).

We examined the time course of behavioral responses to identify whether these goal-directed corrections were learned responses. We found subjects reached the target too quickly in the first flexor perturbation trial in the time-constrained task (hand pushed toward the target; $t_{(9)}=-9.79, p<0.001$ ) and too slowly in the first two extensor perturbation trials (hand pushed away from the target; $\left.t_{(9)}>2.45, p<0.05\right)$. Subjects quickly altered their corrective responses to counter the load and complete their reach within the instructed time (flexion perturbations: Trials $2-10$, all $p>0.16$; extension perturbations: Trials 3-10, all $p>$ $0.14)$. In the time-unconstrained task, subjects took more time to reach the target in the first flexor perturbation trial (hand pushed toward the target; $t_{(9)}=5.12, p=0.001$ ), and less time to reach the target in the first extensor perturbation trial $\left(t_{(9)}<-3.32\right.$, $p=0.009)$. Subjects reduced the strength of their corrective responses by the second perturbation trial, leading to shorter movement times when the flexor perturbation pushed their hand toward the target (Trials 2-10, all $t_{(9)}<1.76, p>0.11$ ) and increased movement times when the extensor perturbation pushed their hand away from the target (Trials 2-10; all $t_{(9)}<$ $0.99, p>0.32$ ). Our results demonstrate that subjects rapidly modified their corrective responses for the timing demands of the task.

Experiment 2: influence of trajectory constraints on rapid feedback responses

Experiment 1 illustrated that seemingly innocuous time demands have a profound impact on how the motor system responds to perturbations during single-joint (Experiment 1A) and multijoint movements (Experiment 1B). We conducted a second experiment to test whether corrective responses enforce trajectory control when the movement path is an explicit goal of the task. We predicted that corrective responses would direct the hand back to the movement trajectory when it was made an explicit goal of the task.

Participants made timed reaching movements to a static target (Fig. 5A; static target), while tracking a moving goal (Fig. 5A; moving target), or maintaining their hand in a constrained path to the spatial goal (Fig. $5 A$; constrained path). We examined corrective responses on random trials by bumping the hand rightward or leftward (lateral to the target) with equal amounts of shoulder and elbow torque (Fig. 5A). This allowed us to test whether elbow feedback responses can produce trajectory control and return the limb to its unperturbed trajectory when the movement trajectory is the explicit goal of the task. 

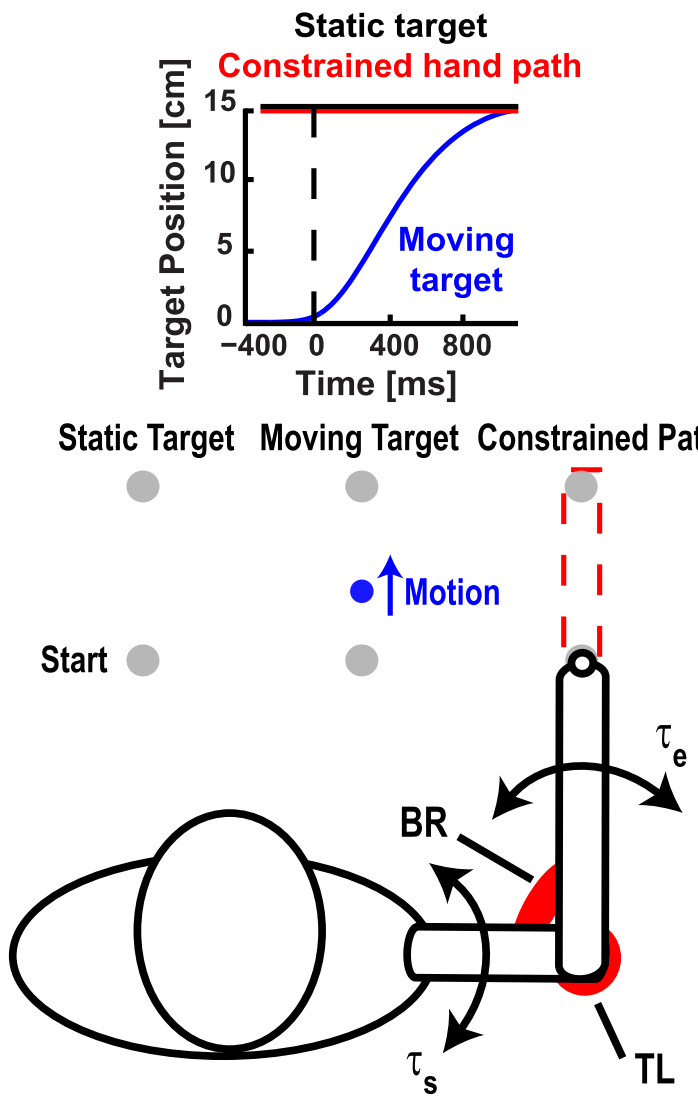

Figure 5. Reaching tasks with different spatial demands. Task and limb configuration used in Experiment 2. Subjects were instructed to reach to a static target (black trace), track a moving target (blue target; arrow indicates target motion), or maintain their hand within a constrained path to the target (dashed red rectangle). The hand was occasionally perturbed rightward or leftward with combined shoulder $\left(\tau_{\mathrm{s}}\right)$ and elbow torques $\left(\tau_{\mathrm{e}}\right)$. Task schematics are separated in space for viewing purposes. Movements were performed from the same initial limb configuration in all tasks.

Figure $6 A-C$ presents hand motion patterns from a typical subject revealing profound changes in corrective responses across the three tasks. The subject moved directly to the static target (i.e., spatial goal) when their arm was disturbed by a perturbation (Fig. 6A), but made a correction to intercept the moving target (Fig. $6 B$ ), or return their hand to the constrained path (Fig. $6 C$ ).

Behavioral differences are further emphasized in Figure 7, which presents perturbation-evoked hand motion from the same exemplar subject shown in Figure 6 (Fig. 7A), as well as group averages (Fig. $7 C$ ). Individual subjects' return times are shown in Figure 7E. Participants made corrections to intercept the moving target or return their hand to the constrained path. The same participants corrected directly to the static target, and this control strategy resulted in a $\sim 150-225 \mathrm{~ms}(20 \%-30 \%)$ increase in the time taken to enter the lateral boundaries of the static goal (Fig. $7 E$; mean \pm SEM: static target $=815 \pm 38 \mathrm{~ms}$, moving target $=$ $651 \pm 37 \mathrm{~ms}$, constrained path $=591 \pm 32$; static vs moving target: $t_{(9)}=5.97, p<0.001$; static target vs constrained path: $\left.t_{(9)}=7.46, p<0.001\right)$.

Individual participants also reduced their lateral hand deviations when the movement trajectory was the explicit task goal (Fig. $7 F$ ). Lateral hand path deviations were largest in the static target task and smallest in the constrained path task (Fig. 7C,F; static target $=3.12 \pm 0.27 \mathrm{~cm}$, constrained path $=1.25 \pm 0.11$; moving target $=1.70 \pm 0.19 \mathrm{~cm}$; static target vs moving target: $t_{(9)}=5.40, p<0.001$; static target vs constrained path: $t_{(9)}=8.29$, $p<0.001$; constrained path vs moving target: $t_{(9)}=2.86, p<$ $0.01)$.

We examined the amplitude of muscle stretch responses to further understand these differences in behavior. Figure 7 shows brachioradialis stretch responses $(\triangle \mathrm{EMG})$ from the same exemplar subject (Fig. 7B) and group data (Fig. 7D). Figure 7 also plots the average amplitude of individual subjects' muscle activity in the LL (Fig. 7G) and VOL time periods (Fig. $7 H$ ). Participants increased their brachioradialis responses in the LL and VOL time windows when the goal was to track the moving target or stay within the constrained path to the goal (Fig. 7D, G; LL: static target vs moving target, $t_{(9)}=2.46, p<0.05$; static target vs constrained path, $t_{(9)}=3.03, p<0.01$; VOL: static vs moving target $t_{(9)}=3.63, p<0.001$; static target vs constrained path, $\left.t_{(9)}=3.01, p<0.01\right)$. We did not observe modulation in the PRE or SL epochs (PRE: static vs moving target: $t_{(9)}=0.46, p=0.67$; static target vs constrained path, $t_{(9)}=0.82, p=0.78$; SL: static vs moving target $t_{(9)}=0.61, p=0.77$; static target vs constrained path, $\left.t_{(9)}=0.58, p=0.85\right)$. ROC analysis demonstrated that goal-directed muscle responses emerged earlier when subjects were instructed to maintain their hand in the constrained movement path ( $~ 65 \mathrm{~ms}$ after perturbation, range $55-72 \mathrm{~ms})$ compared with track the moving target ( $\sim 90 \mathrm{~ms}$, range $70-116 \mathrm{~ms}$; $t_{(9)}=3.74, p<0.01$ ). Our findings indicate that the motor system can indeed behave like a trajectory controller when the movement trajectory is the goal of the task.

Figure 8 presents group data showing lateral hand motion during flexor perturbation trials (Fig. $8 A$ ), as well as individual subjects' average return times (Fig. $8 C$ ) and integrated hand path deviations (Fig. 8D). Subjects again corrected directly to the static target when their limb was disturbed by a perturbation, and this control strategy resulted in an increase in time taken to return to the lateral boundaries of the goal (Fig. $8 A, C$; static vs moving target: $t_{(9)}=3.48, p<0.01$; static target vs constrained path: $\left.t_{(9)}=6.33, p<0.001\right)$. Subjects also reduced their lateral hand deviations when instructed to maintain their hand in the constrained path to the goal target (Fig. $7 A, D ; t_{(9)}=6.72, p<0.001$ ) and track the moving goal $\left(t_{(9)}=5.02, p<0.001\right)$.

Figure 8 also plots group-average lateral triceps responses during flexor perturbation trials (Fig. 8B), as well as the amplitude of individual subjects' average LL responses (Fig. $7 E$ ) and VOL responses (Fig. $8 F$ ). We did not observe significant modulation of muscle responses in the PRE $\left(t_{(9)}<0.7, p=0.45\right)$ or SL epochs $\left(t_{(9)}<1.00, p=0.67\right)$. In contrast, we found an increase in triceps stretch responses in the LL (Fig. $8 B, E$; static vs moving target: $t_{(9)}=1.74, p<0.05$; static target vs constrained path: $t_{(9)}=2.21, p<0.05$ ) and VOL time windows (static vs moving target: $t_{(9)}=1.94, p<0.05$; static target vs constrained path: $\left.t_{(9)}=3.01, p<0.01\right)$. ROC analysis showed that goal-directed muscle responses began earlier in the constrained path task $(\sim 60$ $\mathrm{ms}$ after perturbation, range $=53-73 \mathrm{~ms}$ ) than the moving target task $\left(\sim 75 \mathrm{~ms}\right.$ after perturbation, range $=68-92 \mathrm{~ms} ; t_{(9)}=2.47$, $p<0.05)$.

Finally, we examined the time course of return times and integrated hand-path deviations to determine whether learning processes were required to generate these goal-directed corrections. There were no systematic changes in return times or integrated hand displacements in the static target or constrained path tasks (all $t_{(9)}<1.77, p>0.12$ ). However, in the first trial of the moving target task, we observed larger hand path deviations (extension perturbations: $t_{(9)}=2.21, p=0.037$; flexion perturba- 
A

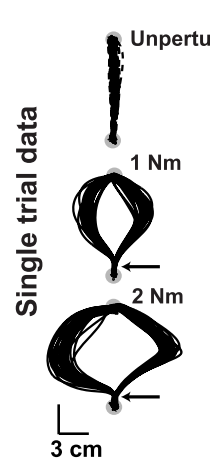

Static Target

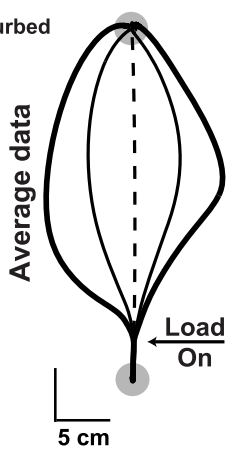

B

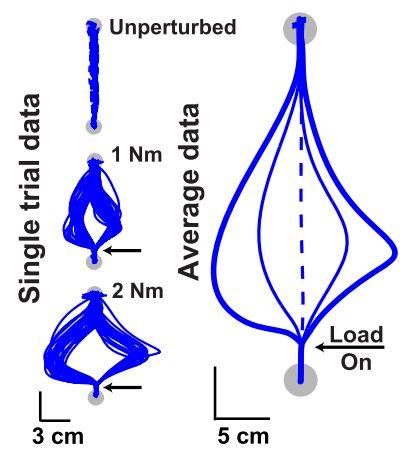

\section{Constrained Hand Path}

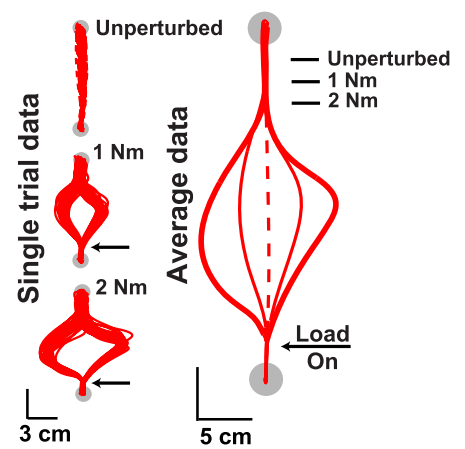

Figure 6. Behavior for reaching tasks with different spatial demands. $A$, Hand paths obtained from a representative subject reaching to the static target. Left, Single trial data. Dashed black traces indicate hand trajectories from unperturbed movements. Solid black traces indicate perturbation trials. Right, Average hand paths obtained from the same typical subject. $\boldsymbol{B}$, Hand paths from the same exemplar subject tracking the moving target. Data are plotted in the same format as in $\boldsymbol{A}$. $\boldsymbol{C}$, Hand paths from the same exemplar subject when instructed to maintain their hand in the constrained path. Data are plotted in the same format as in $\boldsymbol{A}$.

A

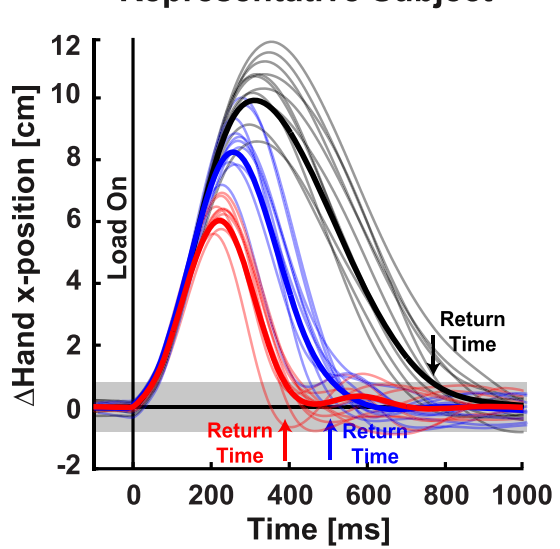

B

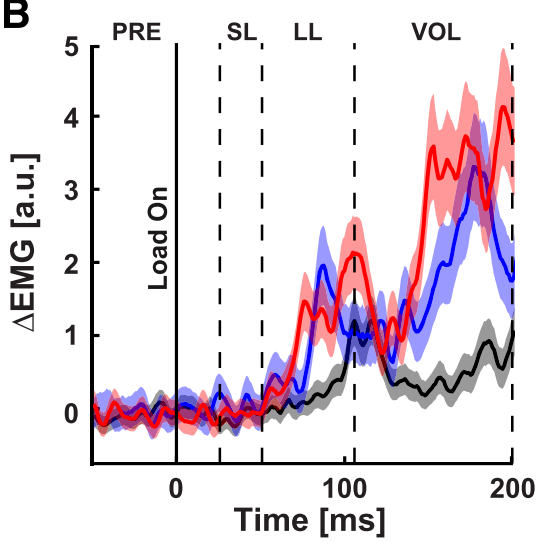

C

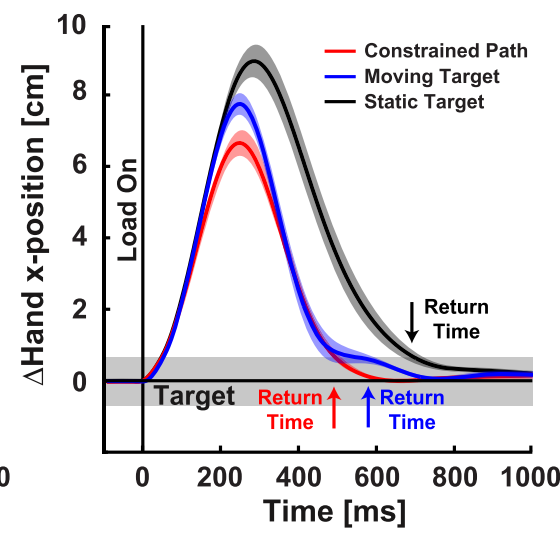

D

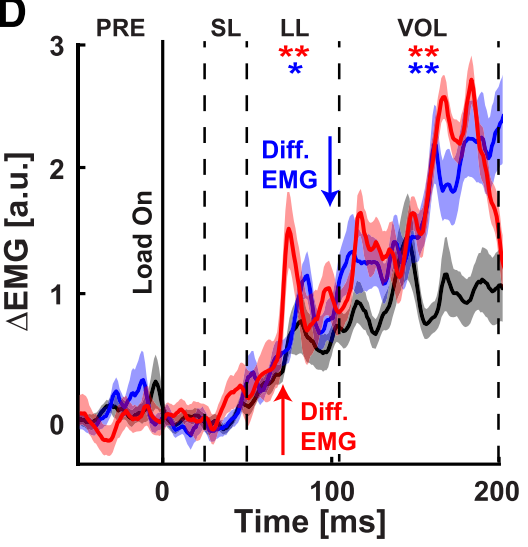

E Individual Subject Averages

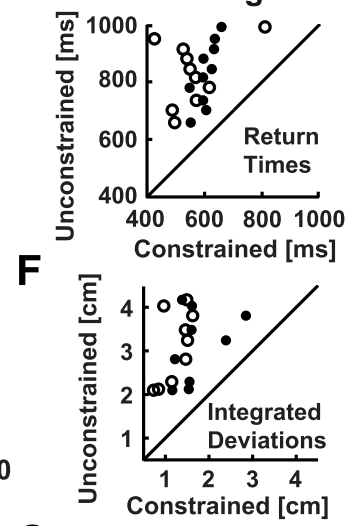

G

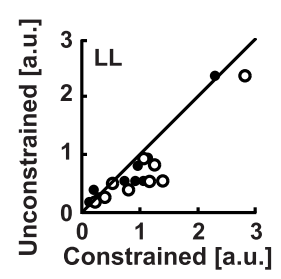

H

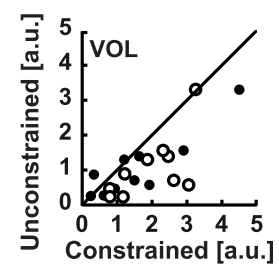

Figure 7. Lateral hand motion and elbow flexor EMG in the trajectory control experiment. $A$, Typical subject data showing lateral ( $x$-axis) hand motion profiles during perturbation trials. Data are aligned to perturbation onset (Load $0 \mathrm{n}$; solid vertical line). Thin lines indicate individual trials ( 10 trials sampled randomly for each condition). Thick lines indicate average hand motion. Shaded gray region represents lateral boundary of the target. Vertical arrows indicate average time the subject took to return to the lateral boundaries of the goal in the constrained path task (red arrow), moving target task (blue arrow), and static target task (black arrow). $\boldsymbol{B}$, Brachioradialis stretch responses from the same exemplar subject. Dashed vertical lines separate different time phases of the muscle stretch response. Data are aligned to perturbation onset (solid vertical line; Load 0 ). Shaded regions represent \pm 1 SEM. C, Group data $(n=10)$ showing lateral hand motion profiles during perturbed trials. Shaded regions represent \pm 1 SEM. Vertical arrows indicate average return times $(n=10)$. Color scheme is the same as in $A$. $D$, Group average brachioradialis stretch responses $(n=$ 10). Red arrow indicates the onset time of separation in muscle activity between the static target and constrained path tasks. Blue arrow indicates the onset time of differentiated muscle activity between the static target and moving target tasks. Asterisks indicate differences between static and moving target tasks, and static target and constrained path tasks. $\boldsymbol{E}$, Return times for perturbed trials in the constrained path versus static target (white dots) and moving target versus static target task (black dots). Data points indicate individual subject averages. $\boldsymbol{F}$, Integrated hand path deviations. Data plotted in the same format as in $\boldsymbol{E}$. G, LL muscle stretch responses. Data plotted in the same format as in $\boldsymbol{E}$. $\boldsymbol{H}$, VOL muscle stretch responses. Data plotted in the same format as in E. ${ }^{*} p<0.05 .{ }^{* *} p<0.01$. 
tions: $\left.t_{(9)}=2.47, p=0.02\right)$ and longer return times (extension perturbations: $t_{(9)}=2.91, p=0.017$; flexion perturbations: $\left.t_{(9)}=-2.21, p=0.041\right)$. Subjects quickly altered their corrective responses, and the effect did not persist past the first trial for return times (Trials 2-10; extension perturbations: all $t_{(9)}<1.25, p>$ 0.24; flexion perturbations: all $t_{(9)}<1.29$, $p>0.21$ ) or hand path deviations (extension perturbations: all $t_{(9)}<1.51, p>$ 0.16 ; flexion perturbations: all $t_{(9)}<1.41$, $p>0.19)$. Collectively, our findings show the motor system rapidly modulates corrective responses based on the goal of the task and can behave like a trajectory controller when the movement trajectory is the goal of the task.

\section{Discussion}

The idea that the motor system uses trajectory control when reaching to a spatial goal is based on the fact that hand motion is relatively straight (Morasso, 1981; Flash and Hogan, 1985) and corrective responses when the limb is mechanically disturbed often return toward the unperturbed trajectory (Bizzi et al., 1982, 1984). Here we found brief reversals in elbow (Experiment 1A) and hand motion (Experiment 1B) when we perturbed the limb during movements with explicit time limits. These brief reversals began within 250 ms of perturbation onset and look like trajectory control because the limb returned toward its unperturbed trajectory. However, when we removed the time constraints, we found that corrective responses were weaker, trajectory reversals disappeared, and subjects moved directly to the spatial goal.

Collectively, these results suggest that our human participants were not controlling a desired limb trajectory but simply avoided attaining the target too quickly (or too slowly depending on the applied load) when they received explicit instructions about the time demands of the task. It is important to note that an implicit timing strategy could also result in reversals in the limb's trajectory. The studies by Bizzi et al. (1982, 1984) examined single-joint elbow movements in monkeys and did not attempt to control the speed of forearm movements. However, the process of training and shaping the animal's motor responses using water reward may have created an implicit timing strategy. The subsequent modeling of this behavior used an implicit timing constraint as movements were completed at a specified time (Hogan, 1984). Admittedly, it is difficult to identify the extent to which implicit timing strategies impact subject behavior. What we show here is that instructions about an explicit timing constraint in human subjects will cause corrections that slow the limb's motion when perturbed toward the goal (or speed up the limb's motion when perturbed away from the goal).

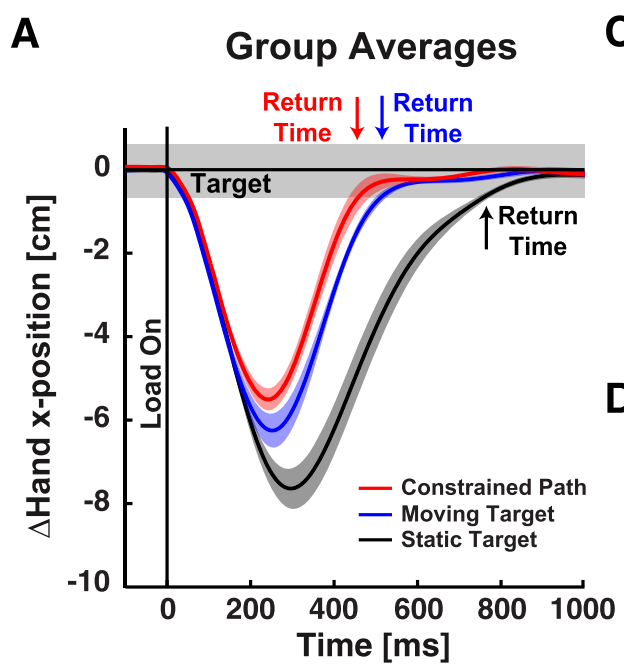

\section{Individual Subject Averages}
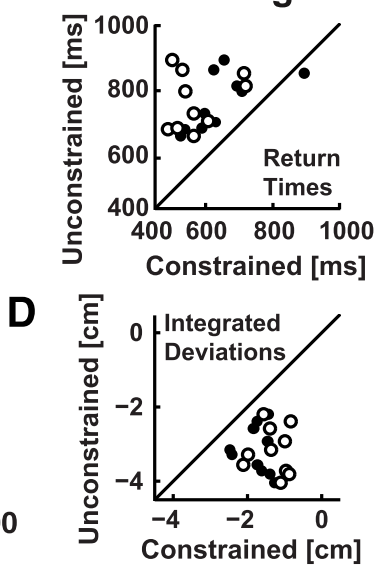

Figure 8. Task, behavior, and elbow extensor EMG in the trajectory control experiment. $\boldsymbol{A}$, Average lateral hand motion profiles during perturbed reaching trials $(n=10)$. Horizontal axis represents the time relative to perturbation onset (Load On; solid vertical line). Shaded curve traces represent \pm 1 SEM. Vertical arrows indicate average return times in the constrained path (red arrow), of time of separation in muscle activity between the static and moving target tasks. Red arrow indicates the onset time of

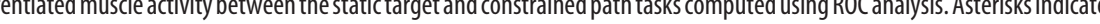
and indicates an individual subject average. $\boldsymbol{D}$, Integrated hand path errors. Data plotted in the same format as in $\boldsymbol{C}$. $\boldsymbol{E}$ $\mathrm{L}$ muscle stretch responses. Data are plotted in the same format as in $\boldsymbol{C} . \boldsymbol{F}, \mathrm{VOL}$ muscle stretch responses. Data are plotted in the same format as in $C^{*} p<0.05 .{ }^{* *} p<0.01$.

Corrective responses when reaching in a force channel have also been interpreted as evidence for trajectory control (Won and Hogan, 1995). In this case, a stiff wall or force channel unexpectedly alters hand motion along a curved path to the goal. Subjects push against the channel, and this applied force has been interpreted as the subject attempting to return to the unperturbed trajectory. However, corrections to the spatial goal would also generate small forces against the virtual wall. These two possibilities (corrections to the trajectory vs the goal) cannot easily be separated based on forces alone as the intended movement in space can only be calculated using a full biomechanical model of the limb.

In our second experiment, we disturbed the limb while subjects reached to a static target or followed a specified trajectory to a spatial goal. A noteworthy finding was that the motor system could perform trajectory control when the limb's trajectory was an explicit goal of the task. These effects are consistent with the 
idea that motor commands map onto the goal when instructed to reach to or hit a target (Todorov and Jordan, 1998) but emphasize spatiotemporal properties of the trajectory when instructed to follow a specified path (Todorov et al., 1997). However, it has been difficult to infer whether feedback from the limb contributes to these distinct control strategies because corrective responses were not measured in these tasks. Here, we disturbed the limb with small mechanical loads and found that corrective responses maintained the limb trajectory when it was the goal but otherwise moved the hand directly to the spatial goal. This flexible control likely extends to other motor tasks where the ability to follow a trajectory dictates how well one performs at the task, such as handwriting, ballet, or a football player chasing an opponent running toward the end zone.

Although we demonstrated that corrective responses are goaldirected and not necessarily toward a desired trajectory, there is compelling evidence that humans initiate relatively straight movements toward a spatial goal, even when it costs more energy (Kistemaker et al., 2010, 2014; Mistry et al., 2013). Previous studies have shown that visual feedback of hand motion can alter movement trajectories, suggesting that we prefer to make visually straight movements (Wolpert et al., 1994, 1995; Flanagan and Rao, 1995). However, straight movements are also observed in subjects that have been blind since early in life (Miall and Haggard, 1995; Sergio and Scott, 1998). Here we show that, even if the nominal trajectory that is initially selected to move to a goal is straight, subsequent corrective responses are goal-directed and not necessarily toward the nominal trajectory.

An important distinction is the difference between a "desired trajectory" and the nominal trajectory specified by an optimal control policy. The reason is that an optimal control policy defines a nominal trajectory that the limb follows when it is unperturbed (Todorov and Jordan, 2002). This nominal trajectory is likely indistinguishable from a desired trajectory until there is a small error or deviation in limb motion (which often occurs). The optimal control policy will make a goal-directed correction, whereas a trajectory control policy will correct back to the original path regardless of the task goal. We have shown that the motor system is capable of a spectrum of goal-directed responses that can resemble trajectory control depending on the requirements of the task.

One possibility is that feedback gains were sufficiently low in our study that corrections were always directed toward the nominal trajectory but were incomplete due to the temporal or spatial constraints of our tasks. However, this is unlikely as subjects increased their feedback responses and returned to their nominal path when instructed to maintain a specific movement trajectory. Subjects can also reduce their corrective responses when they encounter an unexpected load while reaching to an elongated bar versus a small circular target (Nashed et al., 2012). A compelling argument for a general optimal control policy is the observation that, when the limb is perturbed, subjects will veer away from the nominal trajectory to avoid an obstacle in the environment (Nashed et al., 2014). All of these observations, including corrections to a desired trajectory when instructed, highlight that the motor system is best viewed as a general control policy that processes sensory and internal feedback to attain a specific behavioral goal.

We believe that these flexible corrections are achieved through transcortical pathways that transform sensory feedback into goaldirected motor responses (Scott, 2004, 2012). Our results are compatible with evidence that LL responses are altered by features of the task, such as the time urgency to return to a postural target (Dimitriou et al., 2012; Crevecoeur et al., 2013; Omrani et al., 2013), the location of the goal target (Pruszynski et al., 2008,2014 ) and mechanical properties of the limb and environment (Kimura et al., 2006; Kimura and Gomi, 2009; Krutky et al., 2010; Ahmadi-Pajouh et al., 2012; Cluff and Scott, 2013). Here we show that distinct classes of behavioral corrections can be implemented during the LL time period depending on explicit time constraints (Experiments 1A and 1B) or task instructions (Experiment 2).

It is interesting to note that goal-directed muscle responses were slightly delayed $(\sim 15-25 \mathrm{~ms})$ when comparing corrective responses in the constrained path $(\sim 60-65 \mathrm{~ms}$ after perturbation) and moving target tasks ( $\sim 75-90 \mathrm{~ms})$. This finding is surprising given that target motion was predictable. However, predictable yet rapid, time-varying visual stimuli (such as those used in our experiment) can elicit tracking errors that require corrective eye movements (Gardner and Lisberger, 2001; de Brouwer et al., 2002) and hand movements (Miall and Reckess, 2002). The onset of goal-directed responses in our moving target task matches the fastest reported time that visual feedback influences upper limb motor function (75-80 ms) (Gomi, 2008; Pruszynski et al., 2010), suggesting that the motor system may require visual feedback to launch a correction when the target moves quickly or changes velocity.

However, it is unclear what additional information would be gleaned by waiting for visual feedback because only the limb was perturbed in our task. An alternative interpretation is that the delay of goal-directed muscle responses is caused by the time needed to estimate the moving target's future position and update the control policy when a large tracking error occurs during movement. Recent work has shown that subjects can make rapid corrections to avoid an obstacle when their arm is perturbed during reaching ( $\sim 60 \mathrm{~ms}$ after perturbation), but these corrections are delayed when subjects switch to reach a secondary goal in the workspace ( $\sim 75 \mathrm{~ms}$ after perturbation) (Nashed et al., 2014). Thus, time is required to change the control policy from one static goal to another. The present study shows that corrective responses were delayed even further when the motor system was tracking a moving goal. This time delay suggests that the control policy may be dynamic and that vision and proprioceptive feedback may be used to update the control policy when the goal is moving. These results are consistent with the observation that it takes time to update the control policy when a static goal changes position during postural control (Yang et al., 2011) or movement (Dimitriou et al., 2013). Although these studies suggest that it may take the motor system up to $100 \mathrm{~ms}$ to update the control policy, the results of Experiment 2 suggest that the control policy can be updated for a moving goal as quickly as 75-90 $\mathrm{ms}$ after perturbation onset. Whether these differences in response timing reflect different feedback pathways or brain regions remains to be elucidated.

\section{References}

Ahmadi-Pajouh MA, Towhidkhah F, Shadmehr R (2012) Preparing to reach: selecting an adaptive long-latency feedback controller. J Neurosci 32:9537-9545. CrossRef Medline

Archambault PS, Ferrari-Toniolo S, Battaglia-Mayer A (2011) Online control of hand trajectory and evolution of motor intention in the parietofrontal system. J Neurosci 31:742-752. CrossRef Medline

Archambault PS, Ferrari-Toniolo S, Caminiti R, Battaglia-Mayer A (2015) Visually-guided correction of hand reaching movements: the neurophysiological bases in the cerebral cortex. Vision Res 110:244-256. CrossRef Medline

Bizzi E, Accornero N, Chapple W, Hogan N (1982) Arm trajectory formation in monkeys. Exp Brain Res 46:139-143. CrossRef Medline 
Bizzi E, Accornero N, Chapple W, Hogan N (1984) Posture control and trajectory formation during arm movement. J Neurosci 4:2738-2744. Medline

Bryson A, Ho YC (1975) Applied optimal control: optimization, estimation and control. New York: Taylor and Francis.

Caminiti R, Johnson PB, Galli C, Ferraina S, Burnod Y (1991) Making arm movements within different parts of space: the premotor and motor cortical representation of a coordinate system for reaching to visual targets. J Neurosci 11:1182-1197. Medline

Cluff T, Scott SH (2013) Rapid feedback responses correlate with reach adaptation and properties of novel upper limb loads. J Neurosci 33:1590315914. CrossRef Medline

Crevecoeur F, Kurtzer I, Bourke T, Scott SH (2013) Feedback responses rapidly scale with the urgency to correct for external perturbations. J Neurophysiol 110:1323-1332. CrossRef Medline

Crevecoeur F, Cluff T, Scott SH (2014) Computational approaches for goaldirected movement planning and execution. In: The cognitive neurosciences, Ed 5, pp 461-475. Cambridge, MA: Massachusetts Institute of Technology.

de Brouwer S, Yuksel D, Blohm G, Missal M, Lefèvre P (2002) What triggers catch-up saccades during visual tracking? J Neurophysiol 87:1646-1650. Medline

Dickey AS, Amit Y, Hatsopoulos NG (2013) Heterogeneous neural coding of corrective movements in motor cortex. Front Neural Circuits 7:51. CrossRef Medline

Diedrichsen J, Shadmehr R, Ivry RB (2010) The coordination of movement: optimal feedback control and beyond. Trends Cog Sci 14:31-39. CrossRef Medline

Dimitriou M, Franklin DW, Wolpert DM (2012) Task-dependent coordination of rapid bimanual motor responses. J Neurophysiol 107:890-901. CrossRef Medline

Dimitriou M, Wolpert DM, Franklin DW (2013) The temporal evolution of feedback gains rapidly update to task demands. J Neurosci 33:1089810909. CrossRef Medline

Faisal AA, Selen LP, Wolpert DM (2008) Noise in the nervous system. Nat Rev Neurosci 9:292-303. CrossRef Medline

Flanagan JR, Rao AK (1995) Trajectory adaptation to a nonlinear visuomotor transformation: evidence of motion planning in visually perceived space. J Neurophysiol 74:2174-2178. Medline

Flash T, Hogan N (1985) The coordination of arm movements: an experimentally confirmed mathematical model. J Neurosci 5:1688-1703. Medline

Gardner JL, Lisberger SG (2001) Linked target selection for saccadic and smooth pursuit eye movements. J Neurosci 21:2075-2084. Medline

Georgopoulos AP, Schwartz AB, Kettner RE (1986) Neuronal population coding of movement direction. Science 233:1416-1419. CrossRef Medline

Gomi H (2008) Implicit online corrections of reaching movements. Curr Opin Neurobiol 18:558-564. CrossRef Medline

Hatsopoulos NG, Amit Y (2012) Synthesizing complex movement fragment representations from motor cortical ensembles. J Physiol Paris 106: 112-119. CrossRef Medline

Hogan N (1984) An organizing principle for a class of voluntary movements. J Neurosci 4:2745-2754. Medline

Hogan N (1985) The mechanics of multi-joint posture and movement control. Biol Cybern 52:315-331. CrossRef Medline

Kimura T, Gomi H (2009) Temporal development of anticipatory reflex modulation to dynamical interactions during arm movement. J Neurophysiol 102:2220-2231. CrossRef Medline

Kimura T, Haggard P, Gomi H (2006) Transcranial magnetic stimulation over sensorimotor cortex disrupts anticipatory reflex gain modulation for skilled action. J Neurosci 26:9272-9281. Medline

Kistemaker DA, Wong JD, Gribble PL (2010) The central nervous system does not minimize energy cost in arm movements. J Neurophysiol 104: 2985-2994. CrossRef Medline

Kistemaker DA, Wong JD, Gribble PL (2014) The cost of moving optimally: kinematic path selection. J Neurophysiol 112:1815-1824. CrossRef Medline

Krutky MA, Ravichandran VJ, Trumbower RD, Perreault EJ (2010) Interactions between limb and environmental mechanics influence stretch reflex sensitivity in the human arm. J Neurophysiol 103:429-440. CrossRef Medline
Metz CE (1978) Basic principles of ROC analysis. Semin Nucl Med 8:283298. CrossRef Medline

Miall RC, Haggard PN (1995) The curvature of human arm movements in the absence of visual experience. Exp Brain Res 103:421-428. CrossRef Medline

Miall RC, Reckess GZ (2002) The cerebellum and the timing of coordinated eye and hand tracking. Brain Cogn 48:212-226. CrossRef Medline

Mistry M, Theodorou E, Schaal S, Kawato M (2013) Optimal control of reaching includes kinematic constraints. J Neurophysiol 110:1-11. CrossRef Medline

Morasso P (1981) Spatial control of arm movements. Exp Brain Res 42:223227. CrossRef Medline

Mussa-Ivaldi FA (1988) Do neurons in the motor cortex encode movement direction? An alternative hypothesis. Neurosci Lett 91:106-111. CrossRef Medline

Nashed JY, Crevecoeur F, Scott SH (2012) Influence of the behavioral goal and environmental obstacles on rapid feedback responses. J Neurophysiol 108:999-1009. CrossRef Medline

Nashed JY, Crevecoeur F, Scott SH (2014) Rapid online selection between multiple motor plans. J Neurosci 34:1769-1780. CrossRef Medline

Omrani M, Diedrichsen J, Scott SH (2013) Rapid feedback corrections during a bimanual postural task. J Neurophysiol 109:147-161. CrossRef Medline

Pruszynski JA, Kurtzer I, Scott SH (2008) Rapid motor responses are appropriately tuned to the metrics of a visuospatial task. J Neurophysiol 100: 224-238. CrossRef Medline

Pruszynski JA, King GL, Boisse L, Scott SH, Flanagan JR, Munoz DP (2010) Stimulus-locked responses on human arm muscles reveal a rapid neural pathway linking visual input to arm motor output. Eur J Neurosci 32: 1049-1057. CrossRef Medline

Pruszynski JA, Omrani M, Scott SH (2014) Goal-dependent modulation of fast feedback responses in primary motor cortex. J Neurosci 34:4608 4617. CrossRef Medline

Scott SH (1999) Apparatus for measuring and perturbing shoulder and elbow joint positions and torques during reaching. J Neurosci Methods 89:119-127. CrossRef Medline

Scott SH (2004) Optimal feedback control and the neural basis of volitional motor control. Nat Rev Neurosci 5:532-546. CrossRef Medline

Scott SH (2012) The computational and neural basis of voluntary motor control and planning. Trends Cogn Sci 16:541-549. CrossRef Medline

Scott SH, Gribble PL, Graham KM, Cabel DW (2001) Dissociation between hand motion and population vectors from neural activity in motor cortex. Nature 413:161-165. CrossRef Medline

Sergio LE, Scott SH (1998) Hand and joint paths during reaching movements with and without vision. Exp Brain Res 122:157-164. CrossRef Medline

Singh K, Scott SH (2003) A motor learning strategy reflects neural circuitry for limb control. Nat Neurosci 6:399-403. CrossRef Medline

Todorov E (2000) Direct cortical control of muscle activation in voluntary arm movements: a model. Nat Neurosci 3:391-398. CrossRef Medline

Todorov E (2004) Optimality principles in sensorimotor control. Nat Neurosci 7:907-915. CrossRef Medline

Todorov E, Jordan MI (1998) Smoothness maximization along a predefined path accurately predicts the speed profiles of complex arm movements. J Neurophysiol 80:696-714. Medline

Todorov E, Jordan MI (2002) Optimal feedback control as a theory of motor coordination. Nat Neurosci 5:1226-1235. CrossRef Medline

Todorov E, Shadmehr R, Bizzi E (1997) Augmented feedback presented in a virtual environment accelerates learning of a difficult motor task. J Mot Behav 29:147-158. CrossRef Medline

Wolpert DM, Ghahramani Z, Jordan MI (1994) Perceptual distortion contributes to the curvature of human reaching movements. Exp Brain Res 98:153-156. Medline

Wolpert DM, Ghahramani Z, Jordan MI (1995) Are arm trajectories planned in kinematic or dynamic coordinates? An adaptation study. Exp Brain Res 103:460-470. Medline

Won J, Hogan N (1995) Stability properties of human reaching movements. Exp Brain Res 107:125-136. Medline

Yang L, Michaels JA, Pruszynski JA, Scott SH (2011) Rapid motor responses quickly integrate visuospatial task constraints. Exp Brain Res 211:231242. CrossRef Medline 